\title{
A Benign Hamartomatous Tumor - Adenomatoid Odontogenic Tumor
}

\author{
J. Shilpa Rao, B. Balaji Babu, V. Bhavana, B. Jaya Lakshmi \\ Department of Oral Medicine and Radiology, Kamineni Institute of Dental Sciences, Narketpally, Nalgonda, Telangana, India \\ Email for correspondence: juvvadishilpa27@gmail.com
}

\begin{abstract}
Odontogenic tumors are a group of heterogeneous lesions. Among all the tumors adenomatoid odontogenic tumor (AOT) is a benign noninvasive relatively rare which affects younger individuals. In literature, it has been considered as hamartoma rather than a true neoplasm because of its limited size, minimal growth, and lack of recurrence. Hereby, we are discussing about a case of AOT with detailed differential diagnosis, radiographic findings, and treatment.
\end{abstract}

Key words: Adenomatoid odontogenic tumor, adenoameloblastoma, cortical expansion, hamartomatous, hypodense

\section{INTRODUCTION}

Adenomatoid odontogenic tumors (AOT) are considered as a benign, hamartomatous epithelial lesion of odontogenic origin rather than a neoplasia. Steensland first described in 1905; he considered it as a variation of ameloblastoma. ${ }^{[1]}$ Stafne, in year 1948 described it as an "ameloblastic adenomatoid tumor or adenoameloblastoma," since it presented with simulating structures such as canals or glands, he, therefore, described it as an ameloblastoma variant. ${ }^{[2]}$ In 1915 Harbitz referred to it as cystic adantamatoma. In 1969 Philipsen and Birn proposed the current name, i.e., AOT. This name was accepted by the World Health Organization (WHO) in 1971. Nevertheless, in 2005, the WHO defined the histological type of the AOT and defined it as a tumor composed of odontogenic epithelium with a variety of histological patterns within a stroma of mature connective tissue..$^{[3]}$

\section{CASE REPORT}

An 18-year-old female patient visited the Department of Oral Medicine and Radiology with

\section{Quick Response Code Article Info:}

\begin{tabular}{l} 
doi: 10.5866/2017.9.10217 \\
$\begin{array}{l}\text { Received: } 15-12-2017 \\
\text { Revised: } 28-12-2018 \\
\text { Accepted: } 05-01-2018 \\
\text { Available Online: } 15-02-2018,2018 \text { (www. } \\
\text { nacd.in)(C NAD, 2018 - All rights reserved }\end{array}$ \\
\hline
\end{tabular}

a chief complaint of swelling on the middle third of face on the right side since 2 years. The patient was apparently asymptomatic 2 years ago and noticed swelling which was of peanut size initially and slowly increased to present size which is not associated with any symptoms [Figures 1 and 2].

There was no relevant past dental history, medical history, family history, and patient consume mixed diet. The patient brushes once daily with toothbrush and paste and no history of pernicious habit was revealed. On general examination, patient is conscious, cooperative, and comfortably seated in the dental chair. No abnormality was detected in the examination of vital signs.

On extraoral examination, facial asymmetry was seen with swelling on the middle third of face on right side. On intraoral examination of hard tissue missing teeth was seen with respect to 13 and retained deciduous teeth with respect to 53 was seen. On examination of soft tissue, swelling was seen in the labial and buccal vestibule extending from 11 to 17 .

On examination of specific lesion, a solitary diffuse swelling approximately of $3 \times 3 \mathrm{~cm}$ in size was seen on the middle third of face on right side extending anterio-posteriorly from the nasolabial fold to vertical line drawn from the outer canthus of the right eye. Superio-inferiorly it extends from lower orbital margin to horizontal line drawn from the right corner of the mouth. Surface over the 
swelling is smooth. Color is similar to surrounding skin. Intraorally a solitary diffuse swelling approximately of size $3 \times 3 \mathrm{~cm}$ is seen in the right maxillary labial and buccal vestibule extending anterio-posteriorly from 11 to 17 , superio-inferiorly from the vestibule to attached gingiva of 11-17. Surface over swelling is smooth. Color is similar to surrounding mucosa. Displacement of teeth is seen with respect to 12 and 53 .

On palpation, inspectory findings were confirmed. Swelling was non-tender, noncompressible and firm to hard in consistency with soft consistency at 14 with buccolingual cortical expansion with a breach in the continuity was felt with respect to 14 . On aspiration, blood tinged creamish fluid was obtained. Considering the patient's age, sex and site of the swelling and clinical features provisional diagnosis of AOT were made.

Differentialdiagnosis of dentigerouscyst,calcifying odontogenic cyst, ossifying fibroma, KCOT, calcifying odontogenic tumor, and unicystic ameloblastoma was considered. Further investigations were executed.

Radiographic investigations were performed. A maxillary cross-sectional occlusal radiograph was showing a solitary diffuse radiolucency approximately of size $4 \times 2 \mathrm{~cm}$ in the right maxilla extending anterio-posteriorly from interdental areas of 11,12 , and 53 to horizontal line drawn from the occlusal surface of 17 . Mediolaterally from the cervical thirds of 14,15 , and 16 to mid-palatine raphae. An impacted tooth was seen with respect to 13 within the radiolucency. Internal structure was completely radiolucent, and borders are irregular. A buccal cortical expansion was noticed extending from the occlusal surface of 14- to 16. Displacement of 12,53 , and 14 was noticed [Figure 3].

An OPG is showing a solitary diffuse radiolucency approximately of size $4 \times 2 \mathrm{~cm}$ in the right maxilla extending anterio-posteriorly from mid-palatal raphae to the distal surface of distal root of 17. Superio-inferiorly it extends from middle thirds of roots of $12,53,14,15$, and 16 to lower border of right axillary sinus extending till the apical third of root of impacted 13. Internal structure is completely radiolucent with an impacted tooth with respect to 13. Borders are diffuse. Displacement of 12,53 , and 14 was noticed, and multiplanar type of root resorption was seen with respect to 53,14 , and 15 and mesial root of 16 and pushing the lower border of the sinus above [Figure 4].

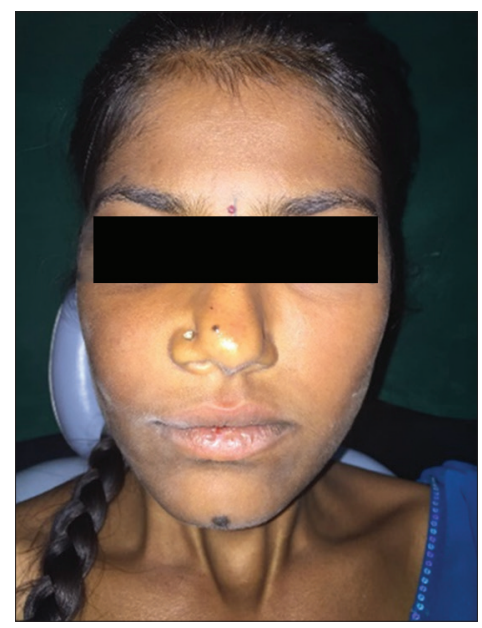

Figure 1: Clinical picture showing extraoral swelling on right middle third of the face

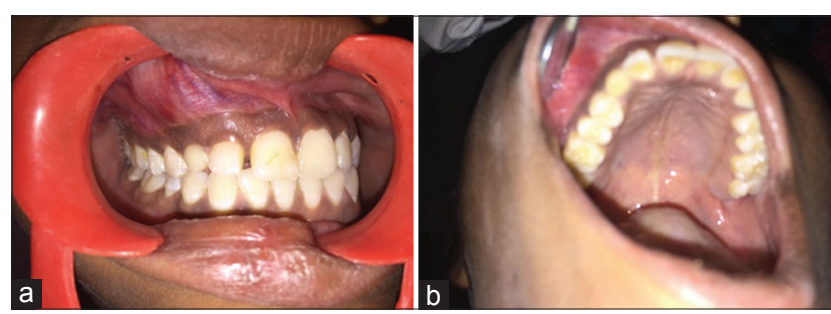

Figure 2: $(a$ and b) Intraoral picture showing swelling in the buccal vestibule and mild swelling on palatal side

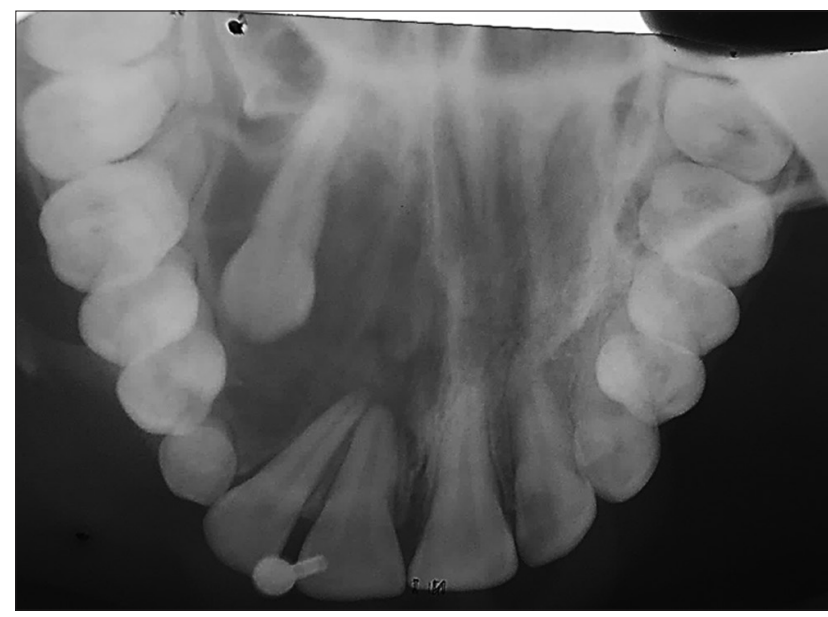

Figure 3: Occlusal radiograph is showing a maxillary crosssectional with a diffuse radiolucent lesion along with an impacted tooth and expansion in the buccal cortical plate

Advanced radiographic examination of CT was done, and axial section shows hypodense area in the maxilla extending anterio-posteriorly on the right side with buccolingual cortical expansion with a 
breach in the buccal cortical plate in the anterior part along with an impacted tooth as hyperdense area and involvement of right maxillary sinus was seen [Figure 5].

Radiological differential diagnosis of adenomatoid odontogenic tumor, dentigerous cyst, calcifying odontogenic cyst KCOT calcifying odontogenic tumor, and unicystic ameloblastoma was considered. The patient was referred to the Department of ENT for complete excision of the lesion was sent for histopathological examination [Figure 6] and is diagnosed as AOT.

\section{DISCUSSION}

AOT is mostly seen in young patients, especially in the second decade of life and is uncommon in patients older than 30 years of age. Females are afflicted by AOT more often than males (2:1). AOT commonly occurs in the maxilla, and the anterior part of the jaw is more frequently involved than the posterior part. ${ }^{[4]}$

Clinically, AOT presents as a slow-growing asymptomatic lesion and is frequently discovered during the routine radiographic examination. The tumors are usually unilocular and $1.5-3 \mathrm{~cm}$ in size, but larger multilocular lesions have been reported in the literature. ${ }^{[5]}$ This tumor manifests as an intraosseous lesion (central type) in the majority of cases (96\%) extraosseous or peripheral lesions account for $<4 \%$ of cases. ${ }^{[6]}$

Intraosseous AOT may be radiographically divided into two types: Follicular (pericoronal) and extrafollicular (extracoronal) types. The former is characterized by a well-defined unilocular radiolucency surrounding the crown and partly the root of an unerupted tooth. The latter is not associated with an unerupted tooth, and the welldefined unilocular radiolucency is found between, above or superimposed on the roots of erupted, permanent teeth. ${ }^{[4,6]}$

The extrafollicular type is also an intra-osseous lesion but not related with an impacted tooth, which accounts for about (24\%) of all the AOT cases, and the peripheral variant is a rare form and is attached to the gingival structures and 18 well-documented cases reported, which accounts for about (3\%). ${ }^{[7]}$ The extraosseous (peripheral/gingival) type of AOT is rarely detected radiographically, but there may be a slight erosion of the underlying alveolar bone cortex. Displacement of neighboring teeth due to tumor expansion is much more common than root resorption. ${ }^{[6]}$

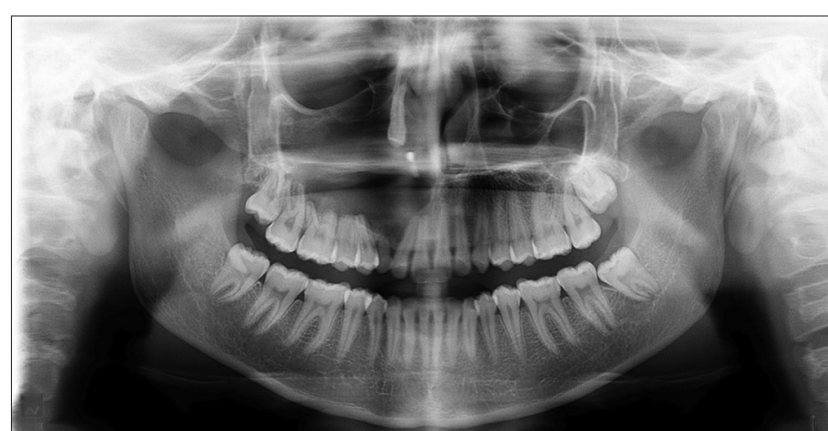

Figure 4: OPG showing a diffuse radiolucent lesion in the maxilla on the right side with an impacted tooth at superior border involving maxillary sinus. Multiplanar pattern of root resorption is seen with respect to $12,53,14,15$, and 16

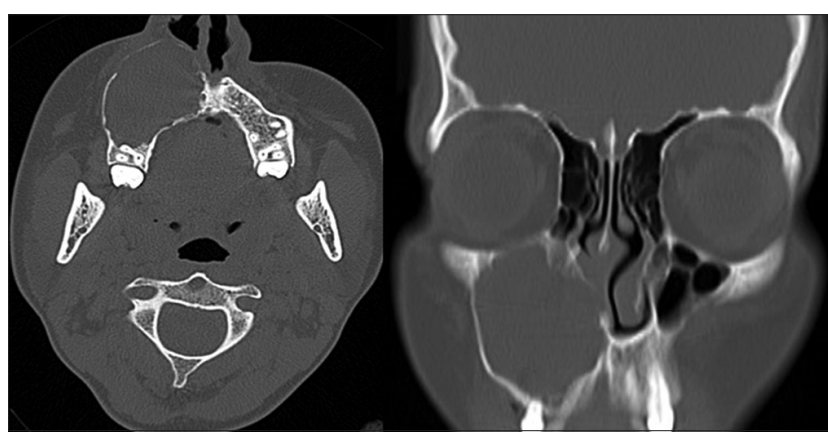

Figure 5: Computed tomography sections show a hypodense lesion in the right maxilla with buccolingual cortical expansion and break in the cortical border in the anterior region associated with an impacted tooth (lesion is surrounding the apical third of root of tooth above cementoenamel junction)

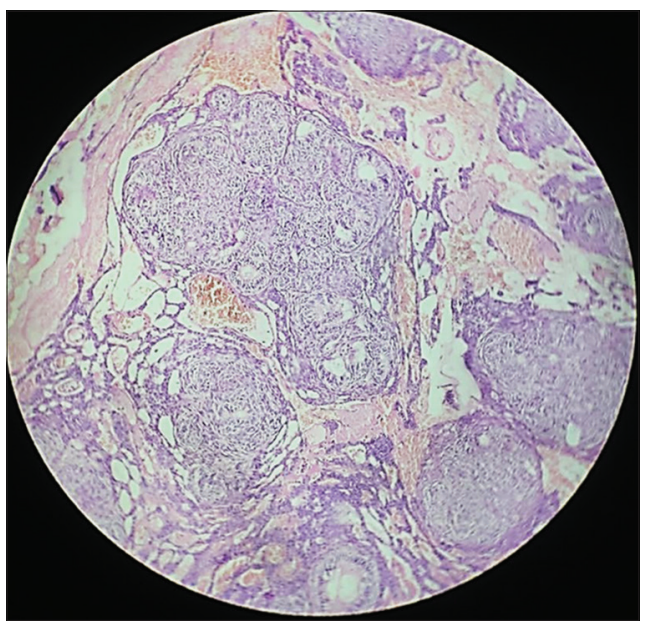

Figure 6: $\mathrm{H}$ and $\mathrm{E}$ section shows cells spindle to oval in shape exhibiting swirling pattern. Some of the cells show lining of ducts or tubular. Cords and trabecular pattern are separating the tumor from cystic structure. Tumor tissue is arranged in nodules and duct 
AOT is frequently misdiagnosed as other odontogenic cysts and tumors on radiographic examination. $77 \%$ of follicular type AOT's are initially diagnosed as dentigerous cysts. Dentigerous cyst encloses only the coronal portion of the impacted tooth with the radiolucency attached at the cementoenamel junction (CEJ), whereas in AOT the radiolucency usually surrounds both the coronal and radicular aspects of the involved tooth.$^{[8]}$ In the present case, the OPG clearly shows the radiolucency covering the crown and extending apical to the CEJ into the cervical third of the root.

Minute radiopacities around the retained tooth can be found in AOT and are considered a characteristic but not a pathognomonic finding. ${ }^{[9]}$ Those calcific deposits are approximately seen in $78 \%$ of AOT. ${ }^{[10]}$ Dare et al. found that intraoral periapical radiographs allow perception of the radiopacities in AOT as discrete foci having a flocculent pattern within radiolucency even with minimal calcified deposits while panoramic often do not. ${ }^{[11]}$ If an AOT has neither an involved tooth nor calcified deposits, the radiographical diagnosis may be enigmatic.

Since all variants show identical benign biological behavior and almost all are encapsulated, conservative surgical enucleation or curettage is the treatment of choice. Recurrence has been reported in very few cases. ${ }^{[12]}$ If the follicle is found during surgery to be uninvolved, it can be easily separated from the tumor, and it will be possible to remove the lesion while leaving the teeth in place, as described by Toida et al. ${ }^{[10]}$

\section{REFERENCES}

1. Ortiz CE, Liceaga EC, Mosqueda TA, Liceaga RR. Tumor odontogenico adenomatoide: Presentacion de un caso de tamano inusual y revision de la literatura. Rev Hos Jua Mex 2006;73:60-3.

2. Lee K, Lee K, Hwang B. Adenomatoid odontogenic tumor: A case report. J Oral Maxillofac Surg 2000;58:1161-4.

3. Garg D, Palaskar S, Shetty VP, Bhushan A. Adenomatoid odontogénico tumor-hamartoma or true neoplasm: A case report. J Oral Sci 2009;51:155-9.

4. Swasdison S, Dhanuthai K, Jainkittivong A, Philipsen HP. Adenomatoid odontogenic tumors: An analysis of 67 cases in a Thai population. Oral Surg Oral Med Oral Pathol Oral Radiol Endod 2008;105:210-5.

5. Philipsen HP, Samman N, Ormiston IW, Wu PC, Reichart PA. Variants of the adenomatoid odontogenic tumor with a note on tumor origin. J Oral Pathol Med 1992;21:348-52.

6. Philipsen HP, Reichart PA. Adenomatoid odontogenic tumor: Facts and figures. Oral Oncol 1998;35:125-31.

7. Philipsen HP, Srisuwan T, Reichart PA. Adenomatoid odontogenic tumor mimicking a periapical (radicular) cyst: A case report. Oral Surg Oral Med Oral Pathol Oral Radiol Endod 2002;94:246-49.

8. Konouchi H, Asaumi J, Yanagi Y, Hisatomi M, Kishi K Adenomatoid odontogenic tumor: Correlation of MRI with histopathological findings. Eur J Radiol 2002;44:19-23.

9. Reichart PA, Philipsen HP. Odontogenic Tumors and Aallied Lesions. London: Quintessence Publications; 2004. p. $105-15$

10. Toida M, Hyodo I, Okuda T, Tatematsu N. Adenomatoid odontogenic tumor: Report of two cases and survey of 126 cases in Japan. J Oral Maxillofac Surg 1990;48:404-8.

11. Dare A, Yamaguchi A, Yoshiki S, Okano T. Limitation of panoramic radiography in diagnosing adenomatoid odontogenic tumors. Oral Surg Oral Med Oral Pathol 1994;77:662-8.

12. Philipsen HP, Reichart PA. The adenomatoid odontogenic tumor: Ultrastructure of tumor cells and non-calcified amorphous masses. J Oral Pathol Med 1996;25:491-6. 